Math. Model. Nat. Phenom.

Vol. 4, No. 4, 2009, pp. 83-102

DOI: $10.1051 / \mathrm{mmnp} / 20094402$

\title{
Stippling the Skin: Generation of Anatomical Periodicity by Reaction-Diffusion Mechanisms
}

\author{
D. J. Headon ${ }^{1}$ *, K.J. Painter ${ }^{2}$ * \\ ${ }^{1}$ The Roslin Institute and Royal (Dick) School of Veterinary Studies \\ University of Edinburgh, Midlothian, EH25 9PS, UK \\ ${ }^{2}$ Department of Mathematics and Maxwell Institute for Mathematical Sciences \\ School of Mathematical and Computer Sciences, Heriot-Watt University, Edinburgh, EH14 4AS, UK
}

\begin{abstract}
During vertebrate development cells acquire different fates depending largely on their location in the embryo. The definition of a cell's developmental fate relies on extensive intercellular communication that produces positional information and ultimately generates an appropriately proportioned anatomy. Here we place reaction-diffusion mechanisms in the context of general concepts regarding the generation of positional information during development and then focus on these mechanisms as parsimonious systems for positioning anatomical structures relative to one another. In particular, we discuss the evidence for reaction-diffusion systems operating in the developing skin to yield the periodic arrangements of hairs and feathers and discuss how best to bring together experimental molecular biology and numerical simulations to yield a more complete understanding of the mechanisms of development and natural variation.
\end{abstract}

Key words: reaction-diffusion, pattern formation, feather, hair, placode

AMS subject classification: $92-02,92 \mathrm{C} 15$

\section{Concepts regarding the origin of positional information in development}

Development is the process by which the complexity of the adult anatomy is generated from the embryo's simplicity. This requires the specialisation of cells into distinct types and that these dif-

*Corresponding authors. E-mail: denis.headon@ roslin.ed.ac.uk, K.J.Painter@ma.hw.ac.uk 

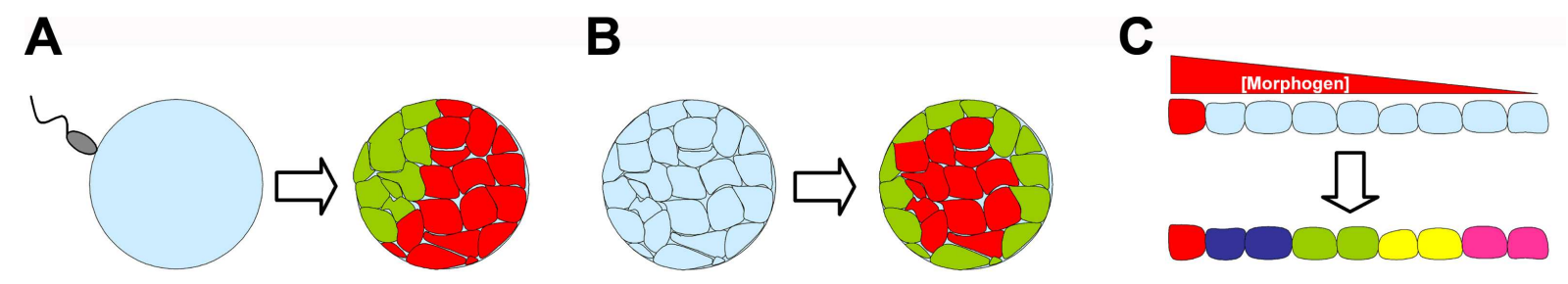

Figure 1: Concepts regarding the origins and elaboration of positional information in development. The different colours denote assumption of distinct cell fates, which are experimentally detectable as changes in gene expression. Light blue indicates naïve cells that have not yet assumed a developmental fate. (A) Generation of asymmetry by sensing of an extrinsic asymmetry, such as sperm entry point upon fertilisation of the egg. (B) Generation of asymmetry through sensing of an intrinsic asymmetry, such as the extent of cell-cell contacts. The schematic shows a bisected embryo. (C) Elaboration of complexity by subdivision of cells in a field by a morphogen gradient emanating from one side of the field (the red cell). The concentration of morphogen across the field is indicated by the red triangle above.

ferent cell types are produced in discrete regions of the embryo to form organs. A fundamental question in developmental biology is, therefore, how the relative position and size of each component of the adult anatomy is determined. In the vertebrate mode of development (known as 'regulative' development) the local environment that an embryonic cell is exposed to is the principal determinant of that cell's fate and thus it is the production, reception and interpretation of intercellular signals that underlies anatomical patterning $[50,16]$.

The cells of the early embryo are developmentally equivalent in that they can generate any cell type of the mature animal. This early symmetry must be broken to begin production of a complex form, necessitating the detection of relative position by cells. A degree of positional information can be gained rather passively by cells sensing either extrinsic asymmetries (e.g. the point of sperm entry at fertilisation or the direction of illumination) or aspects of the intrinsic asymmetry conferred by the embryo's geometry (e.g. when cells at the embryo's periphery are differentiated from those at the core). These cues are used to break the symmetry of the early embryo and generate complexity, but the amount of positional information that can be gained from such means is limited and insufficient to produce a complex anatomy. Once such simple asymmetries have been established, however, they can be used to further subdivide the embryo via production of diffusible signalling molecules, known as morphogens. Morphogens are defined as substances that elicit one of a number of possible effects on cell fate according to their local concentration. Thus, a morphogen gradient produced by one cell population can subdivide a large field of adjacent cells, directing cells along different developmental trajectories according to their proximity to the morphogen's source. These conceptual modes of pattern formation (schematised in Figure 1) have been remarkably successful in explaining aspects of development related to structures that acquire an absolute position in the embryo. In particular, the morphogen gradient as a means to generate positional information has been very influential and has received strong experimental support from systems such as segmentation in insects and digit patterning of the hand $[50,16,48]$. 


\subsection{Absolute versus relative positional information}

In addition to structures that occupy a unique, or a bilateral, position in the body, many organ systems contain elements such as hairs, feathers, renal glomeruli, intestinal villi or pulmonary alveoli that are periodic, being repeated at regular intervals. The widespread nature of periodic structures means that the mechanisms that produce such patterns are of fundamental importance in developmental biology.

The first developmental system that yielded a detailed understanding of periodic patterning was the formation of body segments in the fruitfly Drosophila. In this fly the basic body plan of 14 segments is initially laid out in the embryo as 14 parasegments. The position and identity of the parasegments are ultimately guided by interpretation of morphogen gradients emanating from molecular asymmetries present in the insect egg prior to fertilisation. As a consequence of morphogen gradient interpretation, a set of genes, called pair-rule genes, become expressed in alternating parasegments, constituting a clearly periodic pattern of seven striped elements and seven intervals. In this system there appears to be no unifying patterning mechanism that acts to lay out all of the stripes simultaneously [2], rather, each stripe of pair-rule gene expression is regulated independently of the others [17]. This is most clearly demonstrated by the fact that genetic perturbation of the development of one parasegment does not affect the positioning of the other parasegments in the pattern. Thus each parasegment acquires its location and identity independently of the other elements in the pattern and is defined at an absolute position in the embryo.

The individual placing of each of the 14 parasegment stripes in a regular pattern necessitates a very complex set of molecular interactions and a large number of genes [50]. This absolute positional information model appears unsatisfactory when considering more numerous structures, such as hair follicles, as it is difficult to imagine such bespoke regulatory effort being employed to position every one of the approximately $2 \times 10^{6}$ hair follicles on a human body [39]. A more efficient route for generating a hair pattern would employ a single process to position follicles relative to their neighbours, rather than giving each hair an absolute anatomical location. Such a relative positional system could cover any area of skin with a given density of hairs, whereas if Drosophila were to evolve a 15th parasegment using its 'absolute positional information' system the emergence of an extensive set of new regulatory interactions between genes would be required. Thus a relative positional information system can easily adapt to changes in size and growth and provides a regulatory efficiency compared with absolute positional systems.

\section{Theoretical mechanisms for generating periodic structures in development}

The mechanistic basis for de novo pattern formation in the embryo has long been a source of fascination for both theoretical and experimental biologists, resulting in the proposal of a wide variety of model mechanisms. Broadly speaking, pattern generating mechanisms can be classified according to two principal paradigms, the chemical pre-pattern models and the cell/mechanical- 
interaction models, although there is inevitably a certain blurring between these two classes. In the former, cells are essentially passive, with an underlying molecular network relied on to generate a chemical pre-pattern which determines subsequent cell organization. A number of such mechanisms have been proposed to generate spatially periodic embryonic structures, such as hair follicles, pigment patterns and somites, including the classic Turing/diffusion-driven instability reaction-diffusion model [46], which we describe in greater detail below, and the clock/wavefront model for somitogenesis $[8,6]$.

In cell/mechanical interaction models cells play a fundamental role in driving pattern formation. Chemotaxis models [24] rely on a positive feedback loop in which a population of cells migrates up gradients of a self-generated chemoattractant, resulting in their organization into cell aggregates, as occurs in the life cycle of the cellular slime mold Dictyostelium. Cell adhesion based models rely on graded expression of adhesion molecules to drive the spatial sorting of cell populations [4] while mechanochemical models [32] rely on the reciprocal forces between cells and the surrounding extracellular matrix to generate patterning. All of these cell-based models for patterning are capable of generating periodic structures from an almost uniform initial condition.

\subsection{Reaction-diffusion as a mechanism for generating relative positional in- formation}

The diffusion-driven instability (DDI) mechanism is undoubtedly one of the most compelling paradigms to explain the emergence of periodic patterns from an initially homogeneous condition. This idea, originating in the work of Turing [46] and Gierer and Meinhardt [15], at its simplest entails the existence of two reacting and diffusing substances; an Activator, which both catalyses its own production as well as stimulating the production of its own Inhibitor - this is the 'reaction' (Figure 2). Herein we generally refer to "reaction-diffusion" as an Activator-Inhibitor mechanism capable of generating DDI, although we note that in general this includes, of course, a much wider class of models. When the Inhibitor diffuses more rapidly than the Activator, foci of high Activator production can emerge from near homogeneous initial concentrations of each through a process of short-range activation and long-range inhibition. Limited diffusion of the Activator allows it to locally sustain and intensify the reaction while more rapid diffusion of the long-range Inhibitor acts to dampen it in the surroundings. A simpler variant of this 'Activation-Inhibition' type of reaction-diffusion model is that of 'Substrate Depletion'. Here, no active Inhibitor is required and cells instead undergo patterning through production of a limiting amount of a diffusible Activating factor (the Substrate) and 'competition' for this substance, ultimately leading to the local sequestration and depletion of the ligand by the Activated cells. The appeals of these systems are that the feedback loops are 'closed', so that no outside inputs are required for pattern formation.

Diffusion-driven instability mechanisms therefore amplify deviations from a homogenous state to produce a pattern. Paradoxically, it is the process of diffusion in such systems that destabilises the homogeneous state (hence the name), which resolves itself into foci of high Activator surrounded by an inhibited zone. In order to yield an anatomical outcome, cells in the Activated zone assume one developmental fate while the Inhibited cells assume a different fate. The outcome of this process touches the very core of regulative development - the generation of complexity 
through splitting homogeneous fields of cells into two or more distinct types in a spatially organised manner, without recourse to external positional information. The actual pattern that emerges from the operation of such a system in a 2-dimensional field, whether large or small spots, or stripes, depends on both the kinetics of the reaction and the diffusion characteristics of the Activator and Inhibitor [21, 28] (Figure 2). In addition, the properties of the field within which the mechanism operates can significantly alter the pattern outcome. For example, controlled growth of the underlying field may determine a precise patterning sequence (e.g. [9]), distinct properties at the boundaries may alter the pattern structure (e.g. [12]) and pre-patterning within the field may provide regional heterogeneity prior to the onset of pattern formation. To focus on the properties of the Activation-Inhibition mechanism itself, we will not discuss these aspects here, though we note that they are likely to be important determinants in the patterning of many tissues.

The ability of reaction-diffusion simulations to generate organic-looking patterns and textures has led to their adoption in computer generated imaging of biological entities [47]. Such simulations provide a convenient method to apply naturalistic, non-repeating patterns to images and, akin to the 'regulatory efficiency' that could make such systems useful during embryonic development, relieve graphic designers of the tedium of drawing each pattern element individually. However, while theoretically well developed and successful at mimicking biological patterning, rather few examples of experimentally verified molecular interactions conforming to the predictions of reaction-diffusion models have yet been reported in vertebrate systems [18, 30, 43, 20]. The skin provides an excellent experimental system for exploring periodic patterning in development, as it displays characteristic periodic patterns both in pigmentation (see also article by Othmer et al. in this issue) and anatomical patterning of hair and feather follicles (Figure 3). In addition, simple experiments that have ablated pattern elements [51] or perturbed patterning fields [30] have demonstrated that pigment stripes in fish and hair follicles in mouse are positioned relative to their neighbouring pattern elements and not according to an absolute positional coordinate system, consistent with the operation of a reaction-diffusion mechanism.

\section{Hair and feather follicle development}

Hair and feather fibres grow from follicles, which are cylindrical invaginations in the skin. A glance at intact or plucked skin shows that hairs and feathers are positioned in a periodic manner, raising the question of how this periodicity is generated. Experimental recombination of the two layers of the skin - the epidermis and dermis - indicated that all cells of the embryonic skin are competent to contribute to follicle development, but a patterning process ensures that only some actually do so [42]. The cells that do adopt a follicle fate activate expression of specific genes (Figure 3), pack together to form a structure known as a placode and proliferate rapidly to drive downgrowth into the underlying dermis. Subsequent cell differentiation allows production of a hair fibre, sebum from a sebaceous gland, and a specialised niche for maintenance of stem cells. These complex morphogenetic processes are reviewed elsewhere [41] and here we will focus on the process that apportions placode and interplacode fates in the embryo, which defines the location of follicles in the adult skin. For the purposes of this review, and in line with the majority of spatial modelling, 


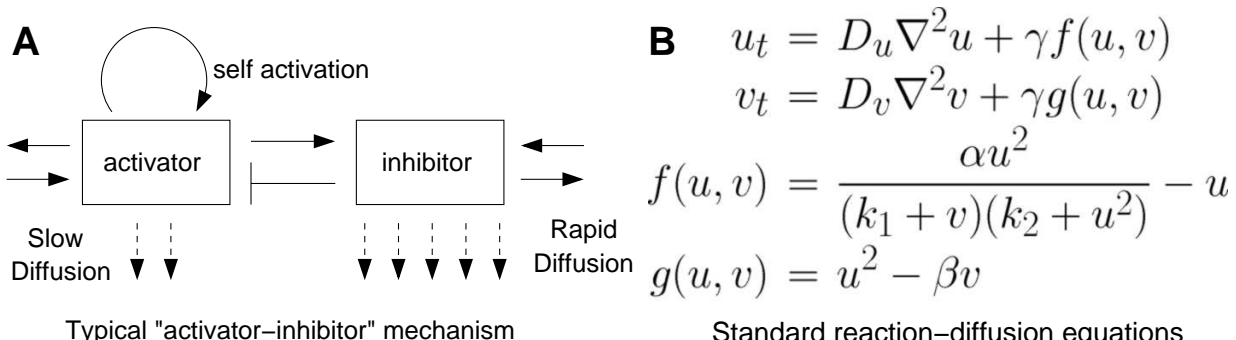

C

(a) Spots
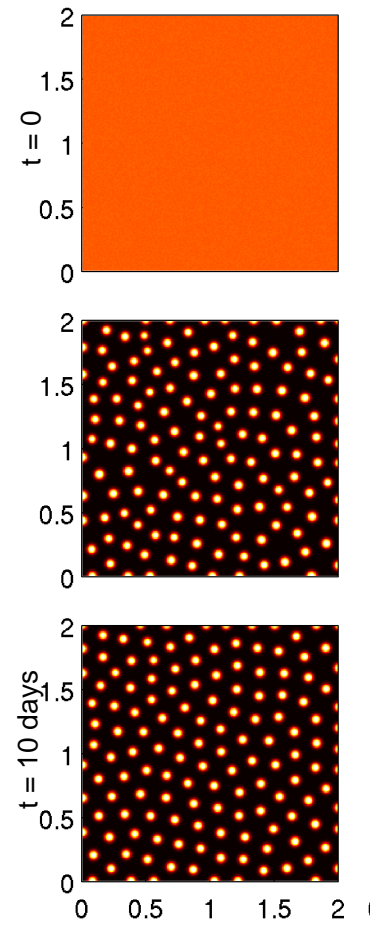

(b) Mixed
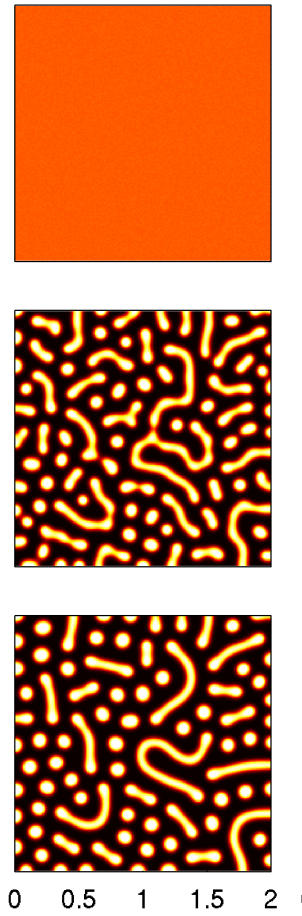

(c) Stripes
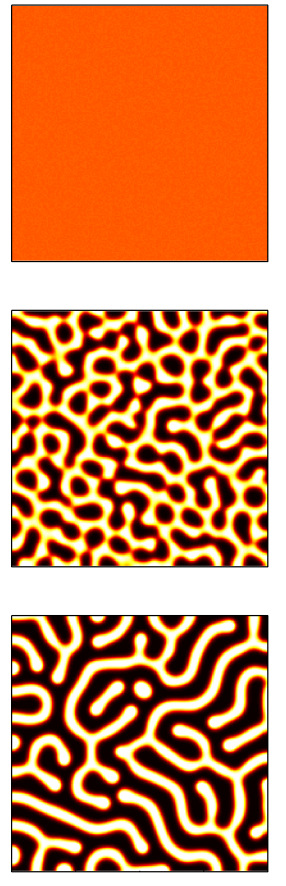

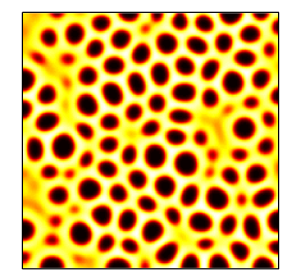

(d) Inverted Spots
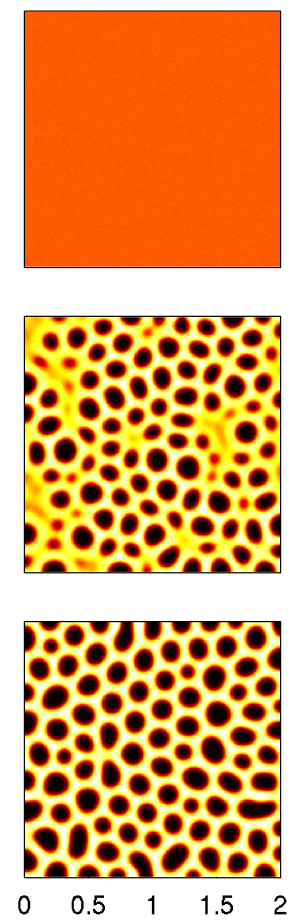

Patterns produced through reaction-diffusion system in B

Figure 2: Reaction-diffusion schematic and simulation. (A) Schematic showing the core features of a pure Activator-Inhibitor system. (B) Mathematical formulation of a two species reactiondiffusion model with Gierer-Meinhardt type kinetics [15]. Here, the Activator and Inhibitor are represented by $u$ and $v$ respectively. The parameters $D_{u}$ and $D_{v}$ represent chemical diffusion coefficients, while $\alpha, \beta, \gamma, k_{1}$ and $k_{2}$ are kinetic parameters. (C) Patterns formed by the reactiondiffusion system given in (B). Plots show Activator profile plotted on a square domain of dimensions 2 by 2 (black to white indicates increasing Activator concentration). For suitable parameters, periodic structures can emerge from an almost homogeneous initial condition to form a variety of 2D patterns (left to right) according to the position in parameter space. For the simulations here, parameters were set at $D_{u}=0.01, D_{v}=1.0, \alpha=1000, \beta=1, \gamma=100, k_{1}=0.01$ and $k_{2}$ varied such that (i) $k_{2}=160$, (ii) $k_{2}=130$, (iii) $k_{2}=110$ and (iv) $k_{2}=90$. Boundary conditions were taken to be zero-flux while initial conditions were set at a small randomized perturbation of the homogeneous steady state. 

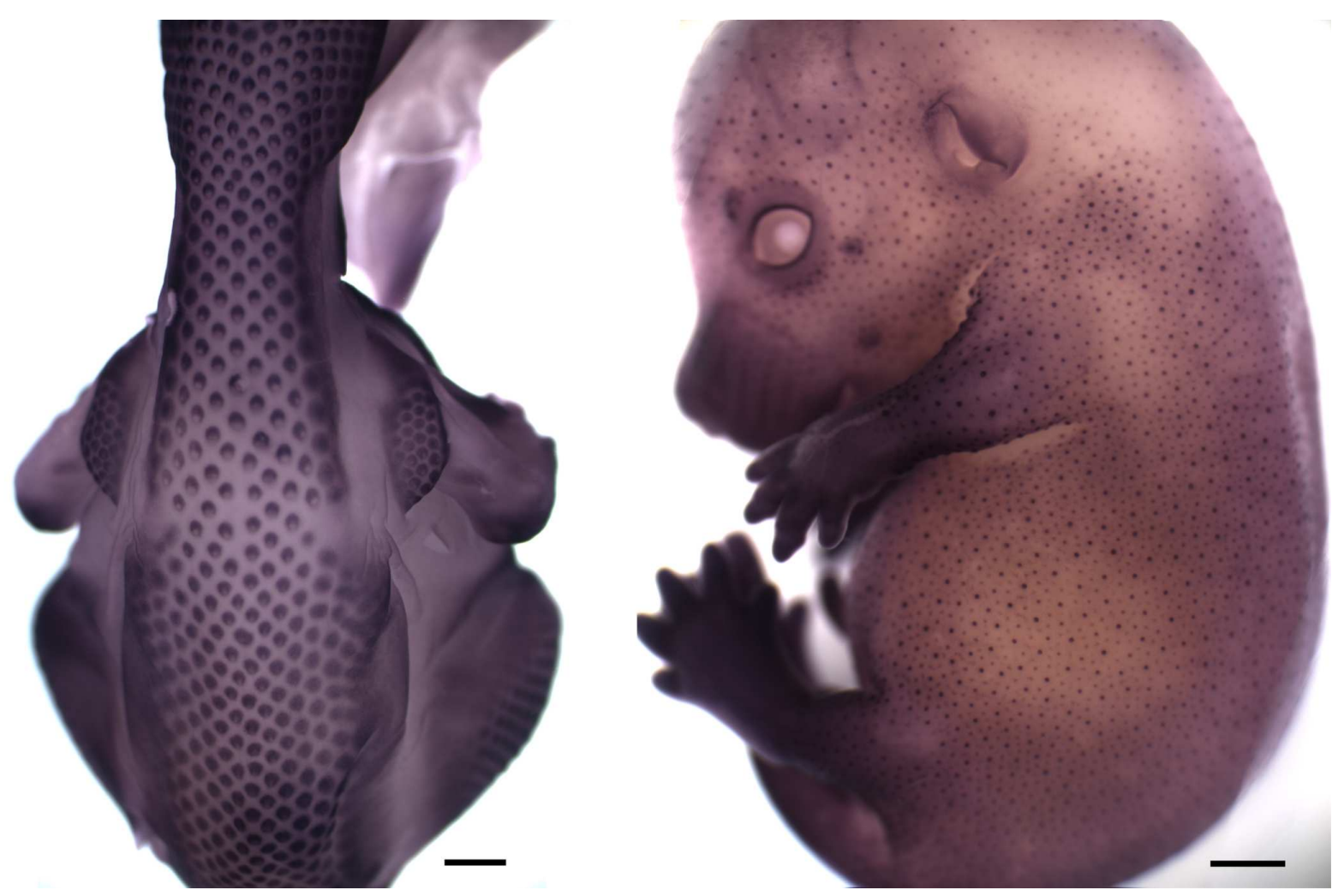

Figure 3: Visualisation of placodes in embryonic chicken (left) and mouse (right) skin by in situ hybridisation to detect $\beta$-catenin gene expression (purple colour). Scale bars indicate $1 \mathrm{~mm}$. Chicken skin produces relatively large placodes that form in a wave that spreads across the skin. Mouse placodes are produced essentially synchronously across the skin.

we will regard pattern formation in the skin as an essentially 2-dimensional problem that occurs only at the epidermal-dermal interface.

\subsection{Identifying the components: Molecular control of hair placode fate}

The last two decades have seen remarkable progress in identifying the molecules that act to control hair and feather placode development. These advances have come from two main approaches; (i) the study of inherited disease conditions affecting skin development and (ii) analyses of candidate genes known to play a role in the development of many organ systems. The wealth of molecular detail available regarding hair [41] and feather [53] development, together with their clearly periodic patterns that can be mimicked by numerical simulations [29, 33], places this field in an ideal position to identify the molecular interactions that govern periodic patterning. In addition, practical experimental considerations including the large amount of embryonic skin tissue available for analysis, the non-essential nature of follicle formation for organism viability, and the ability to culture dissected embryonic skin in order to observe and manipulate pattern formation, support the use of skin as a good model system. 


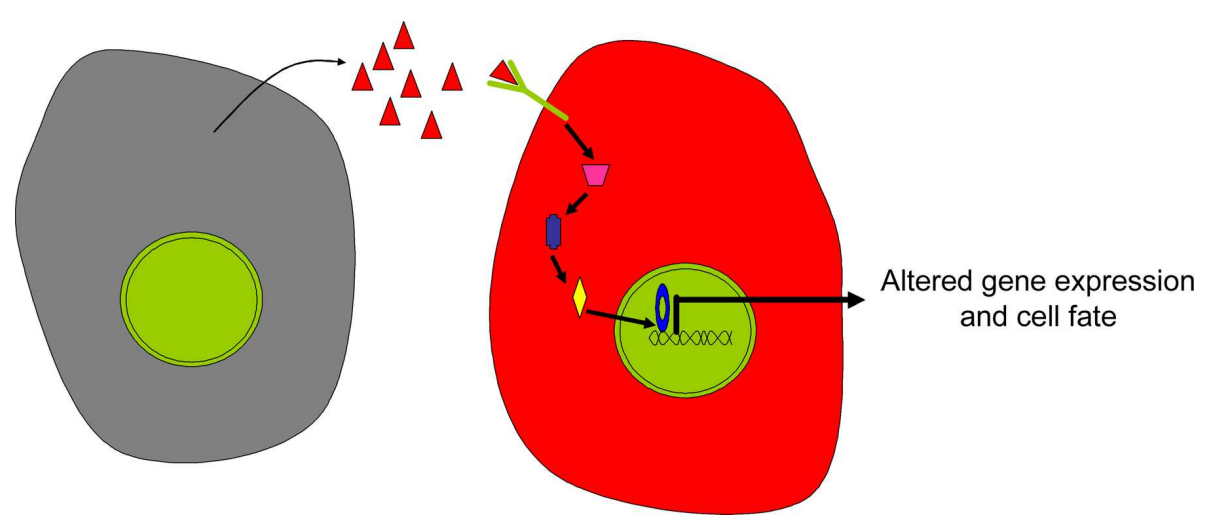

Figure 4: Schematic of cell-cell signalling by protein ligands to illustrate the multi-step nature of intercellular signalling. The signalling cell is in grey, the extracellular signalling molecules are depicted as red triangles. The receiving cell is red, with a transmembrane receptor in green engaging a ligand molecule. Components of the signal transduction machinery of the receiving cell are depicted as coloured shapes.

Several activators and inhibitors of placode formation have been identified, representing candidate Activators and Inhibitors for reaction-diffusion systems. Known activators of placode fate are Wingless/Int family members (Wnts) [34, 3, 35], Ectodysplasin (Eda) [26] and fibroblast growth factors (FGFs) [27, 44]. Suppression of the function of any of these molecules abolishes placode formation, while their experimental activation leads to placode overproduction. Conversely, inhibitors of placode fate have been identified by their ability to block placode formation when activated and to lead to placode overproduction when inhibited. Known inhibitors of placode formation are the bone morphogenetic proteins (BMPs) [22, 36], Dickkopf proteins (Dkks) [3] and epidermal growth factor (EGF) [23,5]. It should be noted that the names given to these proteins reflect the circumstances of their discovery; whether associated with a disease, initially purified from a certain tissue source or first associated with a particular biological activity, and so have a primarily historical significance.

The candidate Activators and Inhibitors listed above are all secreted proteins that act as intercellular signals. The relatively impermeable plasma membrane bounding cells means that reception and interpretation of these macromolecular signals requires a chain of signalling intermediaries, beginning with a transmembrane receptor and typically ending with altered gene expression in the cell nucleus as a primary signal outcome (Figure 4). Thus the Activator and the Inhibitor that appear as individual chemical species in a conceptual reaction-diffusion system (for example, see Figure 2A) are better represented as pathways, with a summation of all activities in the pathway constituting Activator and Inhibitor functions. Though the mechanism of intracellular signal transduction is a subject of intense experimental study, here we will focus on signal production and reception in the extracellular space only, and regard the interpretation of signals by receiving cells as a black box that yields an outcome of altered gene expression or cell fate. 


\section{Putting the pieces together: Molecular interactions corre- sponding to reaction-diffusion systems in hair follicle devel- opment}

When considering the factors within the extracellular milieu that are known to influence placode formation, we are confronted by a complex soup of potential reaction-diffusion Activators (Wnts, FGFs, Eda), Inhibitors (BMPs, EGF), inhibitors of Activators (Dkks) and inhibitors of Inhibitors (Noggin, CTGF, which bind to and inhibit the function of the BMPs in the extracellular space $[7,1])$. While undiscovered molecular activities in follicle development undoubtedly remain to be identified, the fundamental challenge now is to sort through these component parts to identify the key molecular interactions that guide spatial organization of the skin. Here, a basic appreciation of the principles of reaction-diffusion systems can guide us to a number of experimentally testable predictions, though technical considerations make certain features more amenable to study than others. These key characteristics of reaction-diffusion systems are discussed below.

- The basic molecular interactions. The core 'reaction' component of a DDI type reactiondiffusion system indicates that an Activator-Inhibitor system can be characterised by specific molecular interactions: i) the Activator promotes its own activity, and ii) the Activator also indirectly impairs its own function via regulation of an Inhibitor. These interactions are readily testable at the molecular level using standard methods for manipulating intercellular signalling and examining subsequent changes in gene and protein expression. However, feedback loops are common features of cellular signalling networks, in which they act to amplify or dampen signals within signal receiving cells, but not necessarily across a tissue. Thus a determination of these types of regulatory interactions is a necessary step when piecing together a candidate reaction-diffusion system, but further evidence is required to draw firm conclusions.

- Spatial restriction of activity. Detecting patterns of gene expression is a standard technique in developmental biology (see Figure 3), and so it is possible to determine where a given candidate Activator or Inhibitor is produced within a tissue. Simple DDI mechanisms tend to imply specific patterns for Activator and Inhibitor expression, according to the underlying interactions. For a two-component Activator-Inhibitor system of "pure-type", as illustrated in Figure 2A, Activator and Inhibitor production is restricted to the same location; in the case of follicle patterning this would be within the placodes. On the other hand, cross-type Activator-Inhibitor mechanisms produce Activator and Inhibitor distributions out of phase with one another. However, as the process of pattern formation results in cells assuming new fates, which itself leads to the onset of new patterns of gene expression, it is essential to demonstrate whether a candidate Activator or Inhibitor is initially expressed in all cells of a field before undergoing patterning that progressively restricts it to precise spatial locations. Gene expression studies of hair and feather follicle pattern formation have yielded many examples of genes that undergo such spatially dynamic expression, with initially widespread expression becoming restricted to either the placode or the interplacode region. This has 
been termed the 'restrictive' mode of expression [20]. Genes that are expressed only in the placode primordium itself and that are not initially widely expressed are deemed to have a de novo expression pattern and do not play a role in pattern determination.

- Activator and Inhibitor have different ranges of action. To suppress the reaction in the surroundings, the Inhibitor of a reaction-diffusion system must have a greater range of action than the Activator, which is generally taken to mean that the Inhibitor diffuses more rapidly than the Activator. While it might appear that diffusion rates could be predicted from a molecule's molecular weight, it is clear that the transit of protein molecules through live tissues is regulated at the level of interaction with components of the extracellular environment, protein processing, and modifications such as lipid addition to proteins [10, 13, 45]. Thus, diffusion characteristics are not immediately predictable from the physical properties of a particular protein, but instead must be determined empirically in the system under study. For technical reasons it is much easier to detect macromolecules (proteins or RNA) within cells than in the extracellular space and so the diffusion of molecules is difficult to measure accurately at present. A molecule's range of action, however, as opposed to its physical presence, can be determined quite readily by assaying for its effects on gene expression. Indeed, a factor's range of action is a more meaningful property as it encompasses both the diffusion characteristics and the signalling potency of a candidate Activator or Inhibitor. In some circumstances changes in the diffusion properties of a signal may have paradoxical consequences on the signal's range of action. For example, an increase in diffusion rate could result in a signal being diluted below its activity threshold across much of its distribution, resulting in a reduced range of action.

- Timing of molecular interactions relative to pattern appearance. The logic of cause and effect dictates that the molecular interactions that generate a particular pattern must occur before that pattern appears. All interactions, that is, Activator upregulation, induction of Inhibitor production, Inhibitor diffusion and distant inhibition of the Activator, should take place within the time period that the pattern is observed to form from the initial homogeneous state. To perform such analyses an experimental system in which the timing of events can be controlled and synchronised, such as tissues collected and placed in culture, is ideal.

Experimental studies of the molecular control of placode patterning have been led by studies of chicken feather patterning for over a decade. The BMPs were first identified as inhibitors of placode fate assumption in the chick $[22,36]$, in which system was also illustrated most clearly the placode activating functions of the FGFs [27] and Wnt/ $\beta$-catenin [35, 49]. However, the mouse provides distinct advantages over chicken for patterning studies due to the availability of many spontaneous mutations that affect placode formation together with well developed technologies for engineering specific genetic modifications in this species. Two recent studies have used the mouse in an effort to determine whether known placode modulators can be assembled into a system that conforms to reaction-diffusion predictions.

Mou et al. [30] used cultured skin from Eda-deficient mouse embryos to analyse regulatory interactions during primary hair follicle patterning. The application of exogenous proteins, such 


\section{[EDA] (ng/ml)}

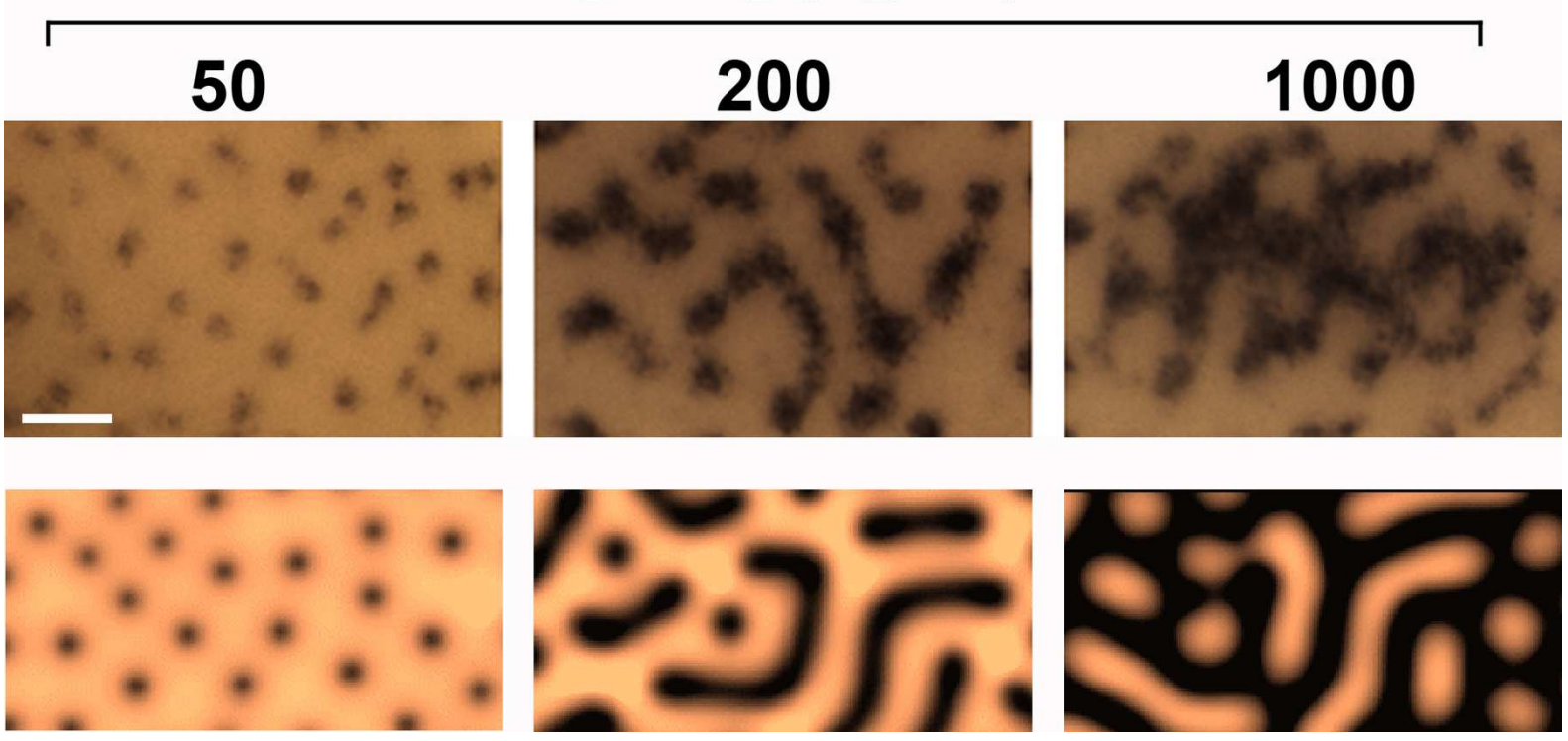

Figure 5: (Top row) The effects of increasing Eda concentration on hair placode formation in cultured mouse skin. Bmp4 gene expression is detected by in situ hybridisation. Application of $50 \mathrm{ng} / \mathrm{ml}$ Eda protein to cultured Eda-deficient skin produces a relatively normal pattern of circular foci, while increasing the concentration of Eda yields fused foci. This experiment was performed according to the method of Mou et al. [20]. Scale bar indicates $200 \mu \mathrm{m}$. (Bottom row) Numerical simulations of the Activator-Inhibitor system given in Figure 2B demonstrate a similar transition on increasing the rate of activator upregulation, $\alpha$; note that alterations to other model parameters are capable of producing similar transitions, c.f. Figure 2C. From left to right we plot the spatial profile for the Inhibitor $(v)$. The equations in Figure 2B were solved numerically on a domain of dimensions $2 \times 2$, with zero flux conditions imposed at the boundaries (for clarity of presentation, only a central rectangular portion of the domain is plotted). Parameters were set at $D_{u}=0.01, D_{v}=1.0, \beta=1, \gamma=100, k_{1}=0.01, k_{2}=150$ with $\alpha$ increased from (left) $\alpha=800$, (centre) $\alpha=1300$ to (right) $\alpha=1600$. Solutions are plotted at $\mathrm{t}=10$ with initial conditions composed of a small random perturbation of the homogeneous steady state. 
as Eda itself, to the cultured skin enabled the synchronization of hair placode patterning and determination of alterations in gene expression and placode arrangement at defined time points in the patterning process. In this model the signalling pathway activated by Eda engagement of its transmembrane receptor Edar was found to represent an Activatory function, with the BMP pathway acting as the Inhibitor. Eda and its transmembrane receptor Edar are expressed homogeneously in the embryonic ectoderm prior to placode formation. As follicle development takes place, Edar expression becomes restricted to placodes, a process that requires local positive autoregulation. BMP family members were identified as rapidly acting inhibitors of Edar gene expression, and Edar was found to induce upregulation of BMP gene expression. As Edar is a transmembrane protein its direct effects are limited to the cells that produce it (i.e. its action is cell autonomous), while BMP responses were found only outside the activated placode regions, demonstrating that in developing skin BMPs act at a distance from their site of synthesis. The lack of BMP responses at their site of synthesis in the placode itself was ascribed to Edar mediated induction of CTGF (connective tissue growth factor), which inhibits the BMPs by binding to them in the extracellular space [52]. This model requires that the range of action of CTGF be less than that of BMP family members. Although a differential range of action was not directly demonstrated in the developing skin, CTGF is known to bind to components of the extracellular matrix [52], which would be predicted to limit its diffusion from its site of synthesis.

Administration of different doses of recombinant Eda and suppression of BMP function allowed extensive manipulation of the spacing between placodes, and the normally circular placodes could be converted into stripes at high concentrations of Eda (Figure 5). As further evidence that Eda and BMP are key regulators of follicle patterning, forced expression of an activated form of Edar that is insensitive to BMP-mediated downregulation caused overproduction of hair follicles in intact animals.

This elucidation of the role of Eda-BMP interactions in determining the hair placode pattern is limited to the spatial arrangement of mouse primary hair follicles. These follicles produce the long guard hairs of the mouse coat, which form earlier in development than the more numerous secondary and tertiary follicles that go on to generate the shorter awl and zig-zag hairs. These other follicle subtypes do not require Eda signalling for their formation as they develop in Eda-deficient animals.

Sick et al. [43] used genetically modified mouse models to address the interplay between the Activatory $\mathrm{Wnt} / \beta$-catenin signalling pathway and the Inhibitory action of Dkks in the patterning of all hair follicle subtypes. $\beta$-catenin is an intracellular protein that regulates expression of other genes by binding to DNA along with partner proteins. $\beta$-catenin is activated by Wnt family proteins, which are diffusible extracellular molecules. The ability of Wnts to activate $\beta$-catenin is suppressed by Dkk family proteins, which inhibit the action of Lrp, a transmembrane receptor for Wnts. Sick et al. found that $\beta$-catenin activity induced expression of $D k k 4$. By modulating Dkk expression in intact animals they were able to reduce the density of hair follicle in the mouse coat and, more importantly, to alter the distribution of hair follicles such that they were clustered together rather than being evenly dispersed. The effects that they observed particularly impacted the positional relationship between primary and secondary hair follicle placodes. They simulated this Activation-Inhibition system across different waves of placode formation and found in silico 
A

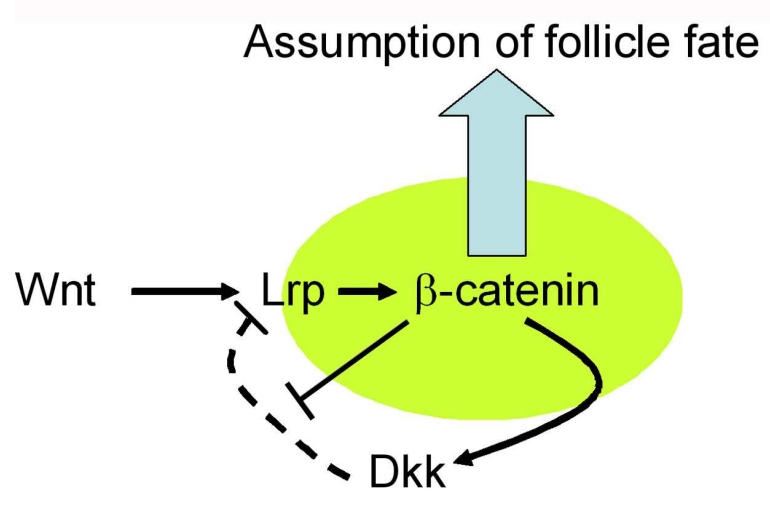

B

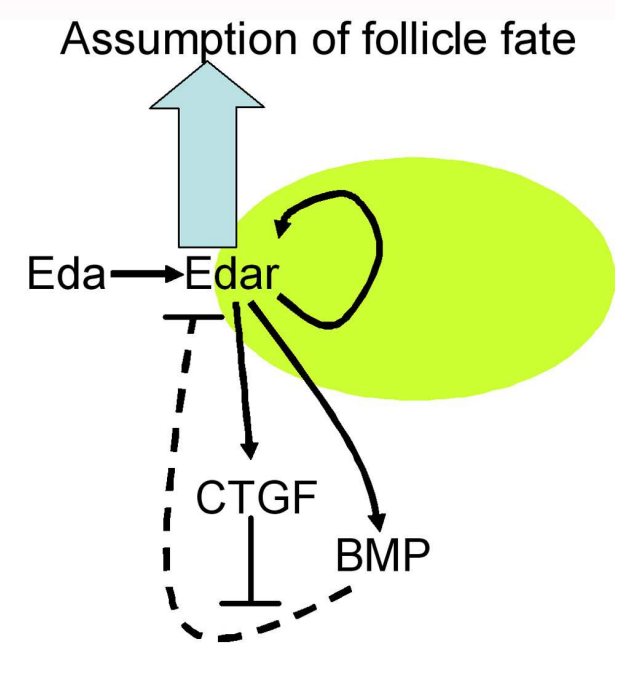

Figure 6: Molecular models for periodic patterning of hair follicles. Cells are indicated as green ovals, with signal receptors (Lrp and Edar) spanning the plasma membrane. A single cell is shown to illustrate the cellular location of the protein species involved in patterning, but this does not indicate that the patterning process is cell autonomous. Dotted lines indicate the component of the model thought to have the greatest range of action. Accumulation of sufficiently high levels of $\beta$-catenin or Edar activity is sufficient to confer a follicular fate on a cell. Protein species indicated by Wnt, Lrp, Dkk and BMP are generic family members; the specific family members (e.g. Dkk4) for mediating these activities are generally unknown. (A) Model proposed by Sick et al. [43]. Local autoactivation of $\beta$-catenin and resistance to the inhibitory effects of Dkk were inferred. Wnt molecules are presumed to have a short range of action and to have a restricted domain of production. (B) Model proposed by Mou et al [30] for primary hair follicle patterning. Note that BMP inhibition of Edar is mediated by transcriptional regulation rather than direct protein-protein interaction. The extracellular ligand, Eda, is produced and diffuses throughout the embryonic skin, and so is not limiting at any location. Activator autoregulation occurs at the level of Eda's transmembrane receptor, Edar, which stimulates its own expression. 
conditions that gave patterns closely matching those engineered in vivo. Accurate simulation of the patterns generated in the mouse skin required that the Activated regions become resistant to the inhibitory action of the Dkks, though a molecular mechanism for this property was not identified. These models for hair follicle pattern formation are schematised in Figure 6.

Though apparently independent of one another, these proposed models describing reactiondiffusion mechanisms in hair follicle patterning should not be regarded as contradictory. Rather, it is more likely that the links between the Eda/BMP and Wnt/Dkk systems (and to FGF and EGF signals) simply remain to be defined. Some resolution of these models comes from the observation that BMPs inhibit $\beta$-catenin action [30] and, at least in chicken skin, Edar and $\beta$ catenin are mutually positively autoregulatory $[11,19]$. Thus several signalling pathways are likely to be resolved into Activatory and Inhibitory networks and, as such, it remains to be determined whether they can be compacted into a simple two component reaction-diffusion model similar to that given in Figure 2B.

A complete understanding of the molecular interactions that direct pattern formation in the skin is clearly some distance away. However, the studies described above, together with a consideration of some basic aspects of molecular biology, highlight several key differences between the simple 2 component reaction-diffusion system (Figure 2) and the types of simulations that would more accurately reflect in vivo patterning processes.

One feature not explicitly present in the conceptual 2-component system is the presence of an inhibitor of the Inhibitor in both models derived from study of hair follicle patterning in mouse (Figure 6). Studies of chicken skin patterning also suggest a key role for this phenomenon [38]. The presence of such an activity would tend to fix a pattern, perhaps when the Activator's activity reaches a threshold point. This fixation of pattern is observed in mouse skin [30] and is likely to be a general feature of organ patterning. However, other reaction-diffusion systems, such as the pigment patterns in fish skin, remain dynamic and unfixed throughout life, so that even in the adult these patterns change in response to pigment cell ablation [51] or growth of the organism [25, 37].

A second difference is the operation of multiple Activators and Inhibitors, each operating through a multi-component pathway. Simulations need not incorporate every component of such pathways, but rather aim to account for the properties of the pathway as a whole. However, it is likely that multiple components will have to be incorporated into models due to the interlocked action of multiple pathways.

Thirdly, the basic cellular processes of transcription and translation required to produce signaling molecules will introduce time delays that are unlikely to be equal for the different molecules and pathways in the system. Simulations incorporating the time delays due to macromolecule synthesis indicate a potentially profound effect on the pattern outcome of reaction-diffusion systems [14].

\section{Moving beyond chemistry}

In the sections above we have focussed our discussion on an essentially chemical process in which cell signalling, gene expression and diffusion combine to form a chemical pre-pattern that is trans- 
lated into distinct cell fates, followed by the initiation of morphogenesis. Conceptually, the separation of 'pattern formation' from subsequent 'morphogenesis' is simple, but might not be respected by biological systems; as remarked on earlier, a number of other models explicitly incorporate morphogenetic processes (such as local cell density, chemotaxis, adhesion and the traction forces exerted by cells) and can directly generate spatial patterning without recourse to a pre-existing chemical pre-pattern. Experimental evidence for such mechanochemical or morphodynamic processes, particularly a role for cell density, has been demonstrated in feather development [20] and theoretical work implies a potentially important role for such physical influences [31, 40]. Experimental studies into the connection between physical processes and pattern formation in development have lagged behind molecular studies primarily due to a lack of techniques to manipulate and measure physical processes in developing tissue, in contrast to the well established methods for measuring and altering gene expression and function. Thus the development of integrated mechanochemical models to explain developmental processes will depend in large measure on the development of suitable bio-engineering methods to enable controlled experimentation.

\section{Outlook: a meeting of experiment and simulation}

It has been known for decades that reaction-diffusion simulations applying hypothetical parameters to hypothetical molecules can accurately mimic follicle spacing patterns [33]. Moving beyond a simple comparison of simulation and anatomy and to a truly synergistic relationship between experimentation and modelling will require, as a first step, the identification of molecular interactions that qualitatively conform to basic experimental predictions, as described above. Once such molecules are identified, it will be necessary to shift focus to a quantitative analysis of their diffusion properties/range of action, the kinetics of the Activation reaction and Inhibition potency, and their production and degradation rates. Such analyses have been avoided by molecular biologists to date, whose focus has generally been on identification of new molecular components of signalling systems, rather than the laborious and unglamorous characterisation of the detailed biochemical properties of known factors. Once determined, these data can be integrated into models to simulate the types of pattern that the interactions focussed on can produce. It is more than likely that initial simulations using such data will fail to mimic accurately the patterns present in nature, indicating the need to incorporate other signals into the system (or possibly inaccuracies in measurement of parameter values). While accurately simulated biological patterns are frequently presented as successes, the real utility of modelling is not to provide comfort but to illustrate gaps in knowledge that require further experiment, ultimately yielding a more complete understanding of the processes under study. Having determined parameter values for biological molecules and performed simulations that yield an approximation of the observed pattern in vivo, the modulation of parameter values in silico can be used to produce characteristic pattern alterations. The experimental goal must be to return to the embryo armed with this knowledge of simulated pattern alterations and test whether reproduction of these altered parameter values in vivo produces a new pattern that matches the simulations.

The blending of in silico and in vivo experimentation described above leads to 2 significant 
problems. The first is that engineering quantitative changes of known magnitude in signalling molecules to match simulated parameter values, particularly for characteristics such as diffusion rates, is not readily achievable with current knowledge. The second problem arises when a given set of molecular interactions is determined to be sufficient to explain an observed biological pattern. At this point it may be tempting to declare the patterning problem 'solved'. However, Occam's razor (in essence "nature does not employ two instruments where one suffices") does not appear to apply in molecular biology. This is likely due to the accidental nature of evolution whereby molecular systems have evolved by random mutation and selection, yielding pathways and mechanisms much more baroque than might be expected from a designed system. Thus molecular redundancy produces a 'completeness problem', whereby it is difficult to know when a full mechanistic description of patterning events and their components has been attained, even though the mechanisms uncovered appear sufficient to account for the observed phenomena.

The most promising, and interesting, approach to address these problems is to exploit the enormous variety of patterns already present in nature. The basic molecular components controlling hair and feather patterning are very similar and so it is likely that modifications of a core skin patterning mechanism, which is currently being defined in laboratory animals, will account for natural pattern variation in a range of vertebrates. Thus, once a basic mechanism has been defined, altered molecular parameters can be sought in nature rather than engineered in the laboratory. In addition, the 'completeness problem' can be addressed by unbiased genetic studies of natural pattern variation, particularly for within-species pattern differences. When a model is 'complete' such genetic studies should highlight only components already incorporated into the model as being responsible for pattern variation, while incomplete models would be subject to the addition of new components as identified. Such an evolutionary-developmental biology (Evo-devo) approach would provide a much deeper understanding of the basic mechanisms of development and also illustrate how the reaction-diffusion system has been modulated during evolution to produce much of the diversity and beauty of the natural world.

\section{Acknowledgements}

We thank Ankita Singal and Chunyan Mou for images used in this manuscript. The authors acknowledge the many studies relevant to theory and experimental examination of hair and feather patterning that have not been cited here due to considerations of space.

\section{References}

[1] J. G. Abreu, N. I. Ketpura, B. Reversade, E. M. De Robertis. Connective-tissue growth factor (CTGF) modulates cell signalling by BMP and TGF-beta. Nat. Cell Biol., 4 (2002), 599-604.

[2] M. Akam. Drosophila development: making stripes inelegantly. Nature, 341 (1989), 282283. 
[3] T. Andl, S. T. Reddy, T. Gaddapara, S. E. Millar. WNT signals are required for the initiation of hair follicle development. Dev. Cell, 2 (2002), 643-653.

[4] N. J. Armstrong, K. J. Painter, J. A. Sherratt. A continuum approach to modelling cell-cell adhesion. J. Theor. Biol., 243 (2006), 98-113.

[5] R. Atit, R. A. Conlon, L. Niswander. EGF signaling patterns the feather array by promoting the interbud fate. Dev. Cell, 4 (2003), 231-240.

[6] R. E. Baker, S. Schnell, P. K. Maini. A clock and wavefront mechanism for somite formation. Dev. Biol., 293 (2006), 116-126.

[7] W. Balemans, W. Van Hul. Extracellular regulation of BMP signaling in vertebrates: a cocktail of modulators. Dev. Biol., 250 (2002), 231-250.

[8] J. Cooke, E. C. Zeeman. A clock and wavefront model for control of the number of repeated structures during animal morphogenesis. J. Theor. Biol., 58 (1976), 455-476.

[9] E. J. Crampin, E. A. Gaffney, P. K. Maini. Reaction and diffusion on growing domains: Scenarios for robust pattern formation. Bull. Math. Biol., 61 (1999), 1093-1120.

[10] Y. Cui, R. Hackenmiller, L. Berg, F. Jean, T. Nakayama, G. Thomas, J. L. Christian. The activity and signaling range of mature BMP-4 is regulated by sequential cleavage at two sites within the prodomain of the precursor. Genes Dev., 15 (2001), 2797-2802.

[11] C. F. Drew, C. M. Lin, T. X. Jiang, G. Blunt, C. Mou, C. M. Chuong, D. J. Headon. The Edar subfamily in feather placode formation. Dev. Biol., 305 (2007), 232-245.

[12] R. Dillon, P. K. Maini, H. G. Othmer Pattern formation in generalised Turing systems. I. Steady state patterns in systems with mixed boundary conditions. J. Math. Biol., 32 (1994), 345-393.

[13] S. Eaton. Release and trafficking of lipid-linked morphogens. Curr. Opin. Genet. Dev., 16 (2006), 17-22.

[14] E. A. Gaffney, N. A. Monk. Gene expression time delays and Turing pattern formation systems. Bull. Math. Biol., 68 (2006), 99-130.

[15] A. Gierer, H. Meinhardt. A theory of biological pattern formation. Kybernetik, 12 (1972), 30-39.

[16] S.F. Gilbert. Developmental biology. Sinauer Associates, Sunderland, 2006.

[17] K. Harding, T. Hoey, R. Warrior, M. Levine. Autoregulatory and gap gene response elements of the even-skipped promoter of Drosophila. EMBO J., 8 (1989), 1205-1212. 
[18] M. P. Harris, S. Williamson, J. F. Fallon, H. Meinhardt, R. O. Prum. Molecular evidence for an activator-inhibitor mechanism in development of embryonic feather branching. Proc. Natl. Acad. Sci. U.S.A., 102 (2005), 11734-11739.

[19] L. Houghton, C. Lindon, B. A. Morgan. The ectodysplasin pathway in feather tract development. Development, 132 (2005), 863-872.

[20] T. X. Jiang, H. S. Jung, R. B. Widelitz, C. M. Chuong. Self-organization of periodic patterns by dissociated feather mesenchymal cells and the regulation of size, number and spacing of primordia. Development, 126 (1999), 4997-5009.

[21] T. X. Jiang, R. B. Widelitz, W. M. Shen, P. Will, D. Y. Wu, C. M. Lin, H. S. Jung, C. M. Chuong. Integument pattern formation involves genetic and epigenetic controls: feather arrays simulated by digital hormone models. Int. J. Dev. Biol., 48 (2004), 117-135.

[22] H. S. Jung, P. H. Francis-West, R. B. Widelitz, T. X. Jiang, S. Ting-Berreth, C. Tickle, L. Wolpert, C. M. Chuong. Local inhibitory action of BMPs and their relationships with activators in feather formation: implications for periodic patterning. Dev. Biol., 196 (1998), $11-23$.

[23] M. Kashiwagi, T. Kuroki, N. Huh. Specific inhibition of hair follicle formation by epidermal growth factor in an organ culture of developing mouse skin. Dev. Biol., 189 (1997), 22-32.

[24] E. F. Keller, L. A. Segel. Initiation of slime mold aggregation viewed as an instability. J. Theor. Biol., 26 (1970), 399-415.

[25] S. Kondo. The reaction-diffusion system: a mechanism for autonomous pattern formation in the animal skin. Genes Cells, 7 (2002), 535-541.

[26] J. Laurikkala, J. Pispa, H. S. Jung, P. Nieminen, M. Mikkola, X. Wang, U. Saarialho-Kere, J. Galceran, R. Grosschedl, I. Thesleff. Regulation of hair follicle development by the TNF signal ectodysplasin and its receptor Edar. Development, 129 (2002), 2541-2553.

[27] M. Mandler, A. Neubüser. FGF signaling is required for initiation of feather placode development. Development, 131 (2004), 3333-3343.

[28] H. Meinhardt. Models for positional signalling with application to the dorsoventral patterning of insects and segregation into different cell types. Development, 107 Suppl (2004), 169180.

[29] J. R. Mooney, B. N. Nagorcka. Spatial patterns produced by a reaction-diffusion system in primary hair follicles. J. Theor. Biol., 115 (1985), 299-317.

[30] C. Mou, B. Jackson, P. Schneider, P. A. Overbeek, D. J. Headon. Generation of the primary hair follicle pattern. Proc. Natl. Acad. Sci. U.S.A., 103 (2006), 9075-9080. 
[31] J. D. Murray. Pattern formation in integrative biology-a marriage of theory and experiment. C. R. Acad. Sci. III, 323 (2000), 5-14.

[32] J. D. Murray. On the mechanochemical theory of biological pattern formation with application to vasculogenesis. C. R. Biol., 326 (2003), 239-252.

[33] B. N. Nagorcka, J. R. Mooney. The role of a reaction-diffusion system in the initiation of primary hair follicles. J. Theor. Biol., 114 (1985), 243-272.

[34] K. Narhi, E. Jarvinen, W. Birchmeier, M. M. Taketo, M. L. Mikkola, I. Thesleff. Sustained epithelial beta-catenin activity induces precocious hair development but disrupts hair follicle down-growth and hair shaft formation. Development, 135 (2008), 1019-1028.

[35] S. Noramly, A. Freeman, B. A. Morgan. beta-catenin signaling can initiate feather bud development. Development, 126 (1999), 3509-3521.

[36] S. Noramly, B. A. Morgan. BMPs mediate lateral inhibition at successive stages in feather tract development. Development, 125 (1998), 3775-3787.

[37] K. J. Painter, P. K. Maini, H. G. Othmer. Stripe formation in juvenile Pomacanthus explained by a generalized turing mechanism with chemotaxis. Proc. Natl. Acad. Sci. U.S.A., 96 (1999), 5549-5554.

[38] K. Patel, H. Makarenkova, H. S. Jung. The role of long range, local and direct signalling molecules during chick feather bud development involving the BMPs, follistatin and the Eph receptor tyrosine kinase Eph-A4. Mech. Dev., 86 (1999), 51-62.

[39] R. Paus, G. Cotsarelis. The biology of hair follicles. N. Engl. J. Med., 341 (1999), 491-497.

[40] I. Salazar-Ciudad, J. Jernvall, S. A. Newman. Mechanisms of pattern formation in development and evolution. Development, 130 (2003), 2027-2037.

[41] R. Schmidt-Ullrich, R. Paus. Molecular principles of hair follicle induction and morphogenesis. Bioessays, 27 (2005), 247-261.

[42] P. Sengel. Morphogenesis of skin. CUP, Cambridge, 1976.

[43] S. Sick, S. Reinker, J. Timmer, T. Schlake. WNT and DKK determine hair follicle spacing through a reaction-diffusion mechanism. Science, 314 (2006), 1447-1450.

[44] H. Song, Y. Wang, P. F. Goetinck. Fibroblast growth factor 2 can replace ectodermal signaling for feather development. Proc. Natl. Acad. Sci. U.S.A., 93 (1996), 10246-10249.

[45] A. A. Teleman, M. Strigini, S. M. Cohen. Shaping morphogen gradients. Cell, 105 (2001), $559-562$.

[46] A.M. Turing. The chemical basis of morphogenesis. Phil. Trans. Roy. Soc. Lond. B, 237 (1952), 37-72. 
[47] G. Turk. Generating textures on arbitrary surfaces using reaction-diffusion. Comp. Graphics, 25 (1991), 289-298.

[48] J.P. Vincent, J. Briscoe. Morphogens. Curr. Biol., 11 (2001), R851-854.

[49] R. B. Widelitz, T. X. Jiang, J. Lu, C. M. Chuong. beta-catenin in epithelial morphogenesis: conversion of part of avian foot scales into feather buds with a mutated beta-catenin. Dev. Biol., 219 (2000), 98-114.

[50] L. Wolpert. Principles of development. OUP, Oxford, 2006.

[51] M. Yamaguchi, E. Yoshimoto, S. Kondo. Pattern regulation in the stripe of zebrafish suggests an underlying dynamic and autonomous mechanism. Proc. Natl. Acad. Sci. U.S.A., 104 (2007), 4790-4793.

[52] K. Yoshida, H. Munakata. Connective tissue growth factor binds to fibronectin through the type I repeat modules and enhances the affinity of fibronectin to fibrin. Biochim. Biophys. Acta, 1770 (2007), 672-680.

[53] M. Yu, Z. Yue, P. Wu, D. Y. Wu, J. A. Mayer, M. Medina, R. B. Widelitz, T. X. Jiang, C. M. Chuong. The biology of feather follicles. Int. J. Dev. Biol., 48 (2004), 181-191. 